\title{
The weight of light
}

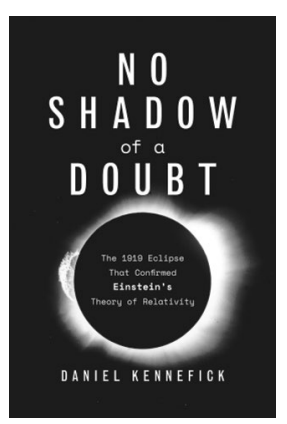

M y lecture notes on general relativity (GR) featured a single, brief chapter on the experimental tests of Albert Einstein's theory: the anomalous perihelion advance of Mercury, the solar redshift and the deflection of starlight. A laconic sentence noted that the first measurement of light deflection was performed by Eddington, Dyson and Davidson during the solar eclipse of 1919. I don't remember being given any more details about these men or their achievements, so I was largely unaware of the drama surrounding the eclipse expeditions of 1919 when I delved into Daniel Kennefick's book on the subject.

Why was the light deflection test so crucial in the early days of GR? The main reason is that until then, the predictive power of GR had not been demonstrated. Mercury's puzzling orbit and the unexplained frequency redshifts of the solar spectral lines preceded Einstein's theory: GR explained both successfully, but it didn't venture onto entirely new ground.

Einstein's prediction for the deflection of starlight passing near the Sun on its way to us combined the assumption that light has mass (which Newton didn't find troubling, unlike many of Eddington's contemporaries) with a bolder insight: gravity alters geometry, hence spacetime is curved. This curvature affects the path of starlight coming close to a massive object like the Sun.

To observe this deflection on Earth, an option is to carry out a comparison of the recorded positions of stars at the limb of the Sun in the latter's presence and absence in the field of view. To image stars so close to a bright source at optical frequencies (on photographic plates, for example), a total solar eclipse can offer ideal conditions. The one of 29 May 1919, with many bright stars and an unusually long duration of totality, represented an opportunity not to be missed. The eclipse expeditions to Sobral and Príncipe became the first successful test of GR (L. C. B. Crispino and D. J. Kennefick, Nat. Phys. 15, 416-419; 2019): the story of those who made it possible, of the difficulties they encountered and of the legacy of these measurements is one worth telling. As an astrophysicist and historian of science who has contributed to the Einstein Papers Project, Kennefick adopts a narrative that develops around three viewpoints: the historic context for GR and its early testing, the conditions and decisions that shaped the measurements of 1919, and the reception and long-lasting consequences of the expeditions' pro-GR results.

Kennefick introduces the protagonists of this scientific adventure as they take up their roles - among them, Frank Watson Dyson, Astronomer Royal and the main organizational drive behind the expeditions; Arthur Stanley Eddington, astronomer with the rare mathematical background necessary to understand GR and present the expeditions' outcome in a meaningful (albeit later contested) manner; and Charles Rundle Davidson, brilliant human computer and unlucky experimenter whose instrument performed so poorly during the eclipse that its data were eventually discarded. Kennefick juggles well the characters in his story, but he seems particularly attached to Eddington and in this sense repeatedly rebuts the accusations of pro-Einstein bias that were thrown at Eddington in the years and decades following the announcement of the expeditions' findings.

Should an author defend so openly one voice in such a collective scientific effort? Kennefick's desire for vindicating Eddington may come across as a little repetitive at times, but it allows him to stress important points too. For example, he emphasizes Eddington's difficult position as a researcher who perceived better than many of his peers the potential revolution ushered in by GR as well as the problematic ambiguity of Newton's theory, which had by then failed physicists and astronomers as a tool. Kennefick also exposes the paradox of how Dyson disappeared from the debate on the validity of the 1919 results even though it was probably he who eventually decided to exclude one instrument's data.

I wonder if condensing the book to fewer than 300 pages might have avoided some repetitions and improved the overall narrative pace; some chapters were re-adapted from previous published works by Kennefick, and that probably didn't help either. However, it isn't the overall length that constitutes the main lack of balance of the book but rather the relative weights assigned to some aspects of the story. While the conditions faced by the two expeditions are described in detail, the values of light deflection found by each team in 1919 are scattered throughout the pages discussing the analysis of the photographic plates; a table summarizing these results along with those given by the 1979 re-reduction of the Sobral data appears about 100 pages from the end of the book.

Kennefick relegates another fascinating point to the final chapters. He observes that physicists expect the precision of a measurement to improve over time. Why did the light deflection measurement provide no satisfaction to later eclipse expedition teams who indeed failed to provide more accurate results? The absence of tangible improvements generated an "experimenters' malaise", which according to Kennefick caused even more scepticism to build up around the 1919 results. How could those men obtain such small errors with sub-optimal weather conditions and far-from-reliable instrumentation? Kennefick stresses the uniqueness of total solar eclipse observations. Eclipses are rare events, and each of them is different; data collection time is very limited, and knowledge transfer across eclipse expeditions can be much more challenging than for laboratory-based experiments. Interestingly, the measurement of the deflection of radio waves by quasars did lead to more accurate values after all.

I wasn't upset that what had been presented to me as an accepted fact in my GR lectures has been the subject of such controversies since 1919, and I'm glad I have a more comprehensive picture now. While Dyson was understandably relieved when the 1922 measurements wiped out "any possible shadow of doubt" about the validity of Einstein's theory, I would argue that scientific doubt is healthy as long as it isn't malicious and operates on the same grounds of rigour and scrutiny as its object.

\section{Reviewed by Gaia Donati}

Associate Editor, Nature.

e-mail: Gaia.Donati@nature.com

Published online: 1 July 2019

https://doi.org/10.1038/s41567-019-0579-4 